\title{
Single-Port Laparoscopic Surgery Using Gelport Access for Cornual Pregnancy
}

Hye Won Kang, M.D.

Department of Obstetrics and Gynecology, Kangbuk Samsung Hospital, Sungkyunkwan University School of Medicine, Seoul, Korea.

Jung Hun Lee, M.D.

Department of Obstetrics and Gynecology, Kangbuk Samsung Hospital, Sungkyunkwan University School of Medicine, Seoul, Korea.

E-mail: mjm.lee@samsung.com

Joong Sub Choi, M.D.

Department of Obstetrics and Gynecology, Kangbuk Samsung Hospital, Sungkyunkwan University School of Medicine, Seoul, Korea.

Jeong Min Eom, M.D.

Department of Obstetrics and Gynecology, Kangbuk Samsung Hospital, Sungkyunkwan University School of Medicine, Seoul, Korea.

(c) Mary Ann Liebert, Inc. DOI: 10.1089/vor.2011.0046
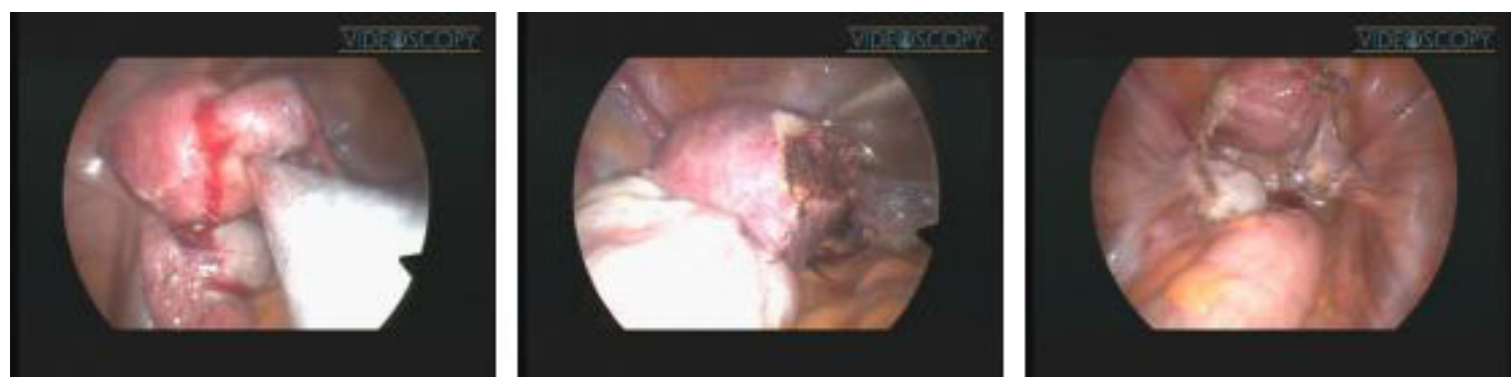

\section{Abstract}

Introduction: Recently, single-port laparoscopic surgery has been studied in various fields and these reports asserted that single-port laparoscopic surgery has cosmetic advantages, shorter recovery, and no short-term or long-term complications compared with the standard laparoscopic approach. ${ }^{1-4}$ We present a video with a running time of 6 minutes 56 seconds of single-port laparoscopic surgery for cornual pregnancy in a 32-year-old nulliparous woman at 5 weeks 5 days gestational age.

Materials and Methods: Operative technique: The patient preparation and port replacement were done in the same way as the surgical technique described in our previous report. ${ }^{3}$ Laparoscopic finding in the pelvic cavity was an intact 2-cm mass in the right cornual portion of the uterus with a thinned-out hyperemic myometrial capsule, adherent to the right adnexa. The remainder of the pelvic structures appeared normal. A dilute solution of vasopressin (10 IU/100 mL normal saline) was injected into the tissue around the base of the cornual ectopic mass to produce vasoconstriction, to reduce the potential for hemorrhage. A transverse elliptical incision was made around the base of the cornual pregnancy with monopolar articulating metzenbaum scissors (Autonomy Laparo-Angle ${ }^{\circledR}$; Cambridge Endo, Inc.,

Framingham, MA). The cornual ectopic mass was excised in a V-shaped manner and the incision was extended laterally to mesosalpinx for salpingectomy. The defect in the myometrial and serosal layers of the cornu was repaired in one layer (1) with interrupted sutures of 1-0 polyglactin 910 (Vicryl ${ }^{\circledR}$; Ethicon, Inc., Somerville, NJ) mounted on a curved needle with a blunt tip, with extracorporeal knots, using a conventional laparoscopic needle holder, and (2) with interrupted sutures of 2-0 polyglactin 910 (Vicryl; Ethicon, Inc.) mounted on a curved needle with a blunt tip, with intracorporeal knots, using articulating 
dissector (Autonomy Laparo-Angle, Cambridge Endo, Inc.) and articulating needle holder. The other tube was excised, and the specimens were removed through the umbilical incision. Following intra-abdominal irrigation, hyaluronic acidcarboxymethylcellulose membrane was coated over the incision sites for preventing postoperative peritoneal adhesions. A drainage tube was inserted through the umbilical incision. After the wound retractor was removed, the incision was dressed with povidoneiodine and closed.

Results and Conclusion: The operating time defined as the period from the incision in the skin to the closure of the skin was 100 minutes, estimated blood loss was $<100 \mathrm{~mL}$, and hemoglobin change between preoperative and postoperative 1 day was $1.5 \mathrm{~g} / \mathrm{dL}$. The drainage tube was removed on postoperative day 2 . The postoperative course of the patient was uneventful. The patient was discharged 3 days after surgery, tolerating a regular diet. Routine follow-up after discharge was performed. Definitive pathologic analysis confirmed the diagnosis of ectopic pregnancy. The surgical wound demonstrated satisfactory cosmetic results. The patient received in vitro fertilization and embryo transfer at postoperative 10 months and has maintained a healthy pregnancy until last follow-up (at 7 weeks 3 days of gestation). We believe that single-port laparoscopic surgery might be an alternative method for cornual pregnancy.

The authors declare that there is no conflict of interest in this research. The authors have no commercial, proprietary, or financial interest in the products or companies described in this article.

Runtime of video: 6 mins 56 secs

\section{Cite this video}

Hye Won Kang, Jung Hun Lee, Joong Sub Choi, Jeong Min Eom, Single-Port Laparoscopic Surgery Using Gelport Access for Cornual Pregnancy, J Laparoendosc Adv Surg Tech Part B Videoscopy. 2011, DOI: 10.1089/vor.2011.0046.

\section{References}

1. Chen YJ, Wang PH, Ocampo EJ, Twu NF, Yen MS, Chao KC. Single-port compared with conventional laparoscopic-assisted vaginal hysterectomy: a randomized controlled trial. Obstet Gynecol 2011;117:906-912.

2. Lee JH, Choi JS, Jeon SW, Son CE, Hong JH, Bae JW. A prospective comparison of single-port laparoscopically assisted vaginal hysterectomy using transumbilical GelPort access and multiport laparoscopically assisted vaginal hysterectomy. Eur J Obstet Gynecol Reprod Biol 2011 [Epub ahead of print]; DOI: 10.1016/j.ejogrb.2011.05.002.

3. Lee JH, Choi JS, Jeon SW, Son CE, Lee SJ, Lee YS. Single-port laparoscopic myomectomy using transumbilical GelPort access. Eur J Obstet Gynecol Reprod Biol 2010;153:81-84.

4. Savaris RF, Cavazzola LT. Ectopic pregnancy: laparoendoscopic single-site surgery-laparoscopic surgery through a single cutaneous incision. Fertil Steril 2009;92:1170.e5-1170.e7.

Original Publication Date: 2011 